\title{
Meropenem in the treatment of complicated skin and soft tissue infections
}

\author{
Douglas N Fish \\ Department of Clinical Pharmacy, \\ University of Colorado Health \\ Sciences Center, Denver, Colorado, \\ USA
}

\begin{abstract}
Meropenem is a broad-spectrum carbapenem antibiotic with excellent activity against many pathogens associated with complicated skin and soft tissue infections (cSSTIs). At least three studies have shown meropenem to have good clinical efficacy and to be well tolerated in the treatment of cSSTIs. Two open-label studies compared meropenem $500 \mathrm{mg}$ every 8 hours (total evaluable $n=146$ ) with imipenem/cilastatin $500 \mathrm{mg}$ every 6 hours $(\mathrm{n}=147)$. Clinical efficacy rates in evaluable patients 7-14 days after end of treatment were similar, $92 \%$ and $100 \%$ in meropenem-treated groups versus $89 \%$ and $100 \%$ in groups receiving imipenem/cilastatin. An additional prospective, randomized, double-blind study evaluated meropenem $500 \mathrm{mg}$ every 8 hours (261 evaluable patients) versus imipenem/cilastatin $500 \mathrm{mg}$ every 8 hours (287 patients). Clinical efficacy rates of meropenem and imipenem/cilastatin $7-28$ days after end of treatment were $86.2 \%$ and $82.9 \%$, respectively. Meropenem was well tolerated in all studies. Carbapenems are currently recommended as appropriate for initial treatment of certain cSSTIs such as those likely to involve mixed and/or multidrug-resistant pathogens. Meropenem is an effective and safe alternative for monotherapy when used for appropriate types of cSSTIs. Higher doses (ie, $1 \mathrm{~g}$ every 8 hours) should be considered for treatment of cSSTIs in higher-risk patients where Pseudomonas aeruginosa is a suspected or documented pathogen.
\end{abstract}

Keywords: meropenem, carbapenems, wound infection, diabetic foot, antibiotics, nosocomial infection

\section{Introduction}

Skin and soft tissue infections (SSTIs) are very frequently encountered in clinical practice and are one of the most common sites of bacterial infections (Nichols 1999; Eron et al 2003; DiNubile and Lipsky 2004). These infections are also among the most common indications for antibiotic therapy and hospital admissions in the US (CDCP 2001). Complicated skin and soft tissue infections (cSSTIs) are those which involve abnormal skin or wounds, occur in a compromised host, or require substantial surgical intervention (DiNubile and Lipsky 2004). Because cSSTIs are by definition more severe in nature and are often associated with complications such as bacteremia and sepsis, patients with these infections are often hospitalized for management with intravenous antibiotics (Nichols 1999; Swartz 2000; Eron et al 2003; Stevens et al 2005). Many cSSTIs are also nosocomial in origin; it has been estimated that these infections are the most common nosocomial infection among surgical patients and occur in approximately $2.6 \%$ of all operations (Wilson 2003).

Meropenem (Merrem ${ }^{\circledR}$, AstraZeneca Pharmaceuticals, Wilmington, Delaware, USA) is a broad-spectrum antibacterial agent of the carbapenem class. Meropenem has excellent activity against a broad range of bacteria including many Gram-positive and Gram-negative pathogens, including many potentially resistant strains such as Pseudomonas aeruginosa, as well as anaerobic organisms (Wiseman et al 1995; Fish and Singletary 1997). Because cSSTIs often involve a diverse range of both aerobic and anaerobic pathogens, meropenem was studied for the treatment of cSSTIs
Correspondence: Douglas N Fish Department of Clinical Pharmacy, University of Colorado Health Sciences Center, 4200 East Ninth Avenue, Campus Box C-238, Denver, Colorado, USA

Tel +I 3033155136

Fax +I 3033154630

Email doug.fish@uchsc.edu 
and was approved by the US Food and Drug Administration (FDA) for this indication in May 2005. The purpose of this article is to briefly review the clinical characteristics and bacterial etiology of cSSTIs, discuss pharmacological and clinical data pertaining to the use of meropenem for these infections, and provide recommendations regarding the role of meropenem in the treatment of cSSTIs.

\section{Complicated skin and soft tissue infections}

\section{Clinical characteristics}

The majority of SSTIs are classified as uncomplicated infections, ie, infections defined as involving only superficial layers of the skin (epidermis, dermis, and subcutaneous tissues) (Eron et al 2003; Stevens et al 2005). Uncomplicated infections such as impetigo, erysipelas, simple abscesses, and simple cellulitis are typically mild to moderate in severity and easily treated with local care with or without oral antibiotic therapy (Eron et al 2003; Raghavan and Linden 2004; Stevens et al 2005). By contrast, cSSTIs involve deeper skin structures such as fascia or muscle layers and require surgical intervention for effective management (Eron et al 2003; Fung et al 2003; DiNubile and Lipsky 2004). These complicated infections are often associated with significant comorbidities such as diabetes mellitus, peripheral vascular disease, critical illness, human immunodeficiency virus (HIV) infection, and other immunocompromised states such as malignancy or posttransplantation (Nichols 1999; Swartz 2000; Fung et al 2003; DiNubile and Lipsky 2004; Stevens et al 2005). Examples of complicated infections include more complex cellulitis and abscesses, perirectal abscesses, post-traumatic or surgical site infections, myositis, necrotizing fasciitis, and infected diabetic and vascular ischemic ulcers. These infections can be challenging to effectively manage because of the great diversity in pathophysiology, microbial etiology, clinical manifestations, severity, and potential for additional complications such as bacteremia, sepsis syndrome, and organ dysfunction (Swartz 2000; Eron et al 2003; Lipsky et al 2004; Stevens et al 2005).

The pathophysiology of cSSTIs is also significant in terms of potential effects on antimicrobial efficacy. Since the efficacy of antimicrobials depends on the ability to achieve adequate concentrations at a particular site of infection, good distribution to infected tissues is a key feature of any agent. However, cSSTIs are commonly associated with vascular insufficiency due to underlying comorbidities, disruption of normal blood and lymphatic flows, and/or areas of devitalized tissue, all of which may severely limit the penetration of drugs to the site of bacterial infection. The possibility of significantly decreased penetration of antimicrobials to tissues involved in cSSTIs thus may potentially lead to inadequate drug concentrations, clinical and microbiological treatment failure, and the development of resistance among bacterial pathogens. Successful antimicrobial management of cSSTIs therefore depends on the use of agents possessing the combination of good intrinsic activity against potential pathogens and intrinsic ability to distribute to tissues of the skin and skin structures, plus use of relatively high doses which increase the likelihood of sufficient tissue penetration.

\section{Bacterial etiology}

Complicated SSTIs are associated with a broad range of potential pathogens (Table 1) (Doern et al 1999; Jones et al 1999, 2003; Bowler et al 2001; Fung et al 2003; Rennie et al 2003; Jodrá et al 2006). Aerobic Gram-positive cocci are the predominant pathogens in most types of cSSTIs, particularly those that are community-acquired in origin. Staphylococcus aureus, other staphylococci, and various

Table I Bacterial pathogens associated with complicated skin and soft tissue infections (Doern et al 1999; Jones et al 1999, 2003; Bowler et al 200 I; Fung et al 2003; Rennie et al 2003; Jodrá et al 2006)

\begin{tabular}{|c|c|c|}
\hline \multirow[b]{2}{*}{ Organism } & \multicolumn{2}{|c|}{ Frequency of isolation } \\
\hline & $\begin{array}{l}\text { All } \\
\text { infections } \\
(\%)^{a}\end{array}$ & $\begin{array}{l}\text { Hospital- } \\
\text { acquired } \\
\text { infections (\%) }\end{array}$ \\
\hline Gram-positive aerobes & $57-60$ & $29-55$ \\
\hline Staphylococcus aureus (all) & $29-46$ & $13-19$ \\
\hline Staphylococcus aureus (MR) & $3-15$ & $8-12$ \\
\hline Coagulase-negative staphylococci & $3-5$ & $6-13$ \\
\hline Streptococci & $7-20$ & $3-4$ \\
\hline Enterococcus spp. & $3-8$ & $5-14$ \\
\hline Gram-negative aerobes & $22-26$ & $25-47$ \\
\hline Pseudomonas aeruginosa & $3-11$ & $3-9$ \\
\hline Escherichia coli & $4-13$ & $7-12$ \\
\hline Enterobacter spp. & $5-6$ & $4-9$ \\
\hline Klebsiella pneumoniae & $3-4$ & $4-6$ \\
\hline Proteus spp. & $4-6$ & $2-4$ \\
\hline Acinetobacter spp. & ND & $3-4$ \\
\hline Serratia spp. & $2-3$ & $\mathrm{I}-4$ \\
\hline Anaerobes & $17-33$ & ND \\
\hline Bacteroides spp. & $5-13$ & ND \\
\hline Peptostreptococcus spp. & $4-8$ & ND \\
\hline Prevotella spp. & 3 & ND \\
\hline Clostridium spp. & I & ND \\
\hline
\end{tabular}

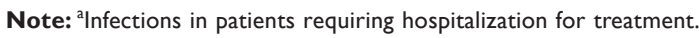
Abbreviations: MR, methicillin-resistant; ND, no data reported. 
streptococci (particularly of groups A and B) are the most common organisms (Bowler et al 2001; Fung et al 2003; Raghavan and Linden 2004). The incidence of infections caused by methicillin-resistant $S$. aureus (MRSA) has been steadily increasing; MRSA now accounts for approximately $60 \%$ of all $S$. aureus isolates from nosocomial infections in the US (DPH 2004). Even more alarming is the growing incidence of infections due to community-acquired MRSA. Although MRSA has been traditionally regarded as a hospital-acquired pathogen, this organism has also emerged as a common cause of community-acquired infections with approximately $30 \%$ of all MRSA isolates now communityacquired in origin (Burkharie et al 2001; Eady and Cove 2003). A recent study of MRSA infections in three geographic areas within the US reported that $17 \%$ of all isolates were community-acquired in origin (Fridkin et al 2005). This study also found that $77 \%$ of these infections involved skin and soft tissue, and the overall incidence of community-acquired MRSA infections was significantly higher among persons less than two years of age. Specifically in cSSTIs, MRSA has been reported in up to $10 \%-15 \%$ of patients overall, and $5 \%-15 \%$ of patients with nosocomial infections (Jones et al 1999, 2003; Fung et al 2003; Rennie et al 2003; DPH 2004; Raghavan and Linden 2004). Enterococci are also common pathogens in cSSTIs and are isolated with somewhat greater frequency in nosocomial infections (Jones et al 1999, 2003; Bowler et al 2001; Raghavan and Linden 2004). Up to $20 \%$ of enterococci isolated from these infections are now vancomycin-resistant (VRE) (Rennie et al 2003; Raghavan and Linden 2004). S. aureus, enterococci, and coagulasenegative staphylococci are particularly important pathogens in surgical wound infections and together account for approximately $46 \%$ of all surgical site infections; both MRSA and VRE occur more frequently in these infections as well (DiNubile and Lipsky 2004; DPH 2004).

Gram-negative organisms which are frequently seen in cSSTIs include the Enterobacteriaceae (particularly Escherichia coli, Enterobacter spp., Klebsiella spp., and Proteus spp.) and P. aeruginosa (Jones et al 1999, 2003; Swartz 2000; Fung et al 2003; Rennie et al 2003; Stevens et al 2005). Complicated skin and soft tissue infections commonly associated with $P$. aeruginosa include lower extremity infections (particularly in patients with comorbidities such as vascular insufficiency or diabetes), surgical wound infections, chronic renal disease, and diabetes or other immunocompromising diseases (Bowler et al 2001; Pellizer et al 2001; Eron et al 2003; Rennie et al
2003; DPH 2004; Lipsky et al 2004). Of particular concern is the rapid spread of resistance mediated by extendedspectrum $\beta$-lactamases (ESBLs) among organisms such as Klebsiella pneumoniae and Escherichia coli. Organisms that produce ESBLs are usually resistant to multiple antimicrobials including third-generation (eg, ceftriaxone, cefotaxime, ceftazidime) and fourth-generation (ie, cefepime) cephalosporins and aztreonam (Bush 2001; Paterson et al 2003), and are also associated with high rates of resistance to aminoglycosides and fluoroquinolones (Paterson et al 2000, 2003). Although recent data for cSSTIs are not readily available, a recent international study of Gram-negative bacilli isolated from intra-abdominal infections found $7 \%$ of E. coli, $13 \%$ of Klebsiella spp., and $18 \%$ of Enterobacter spp. were ESBL producers (Chow et al 2006). These data are consistent with data reported specifically from the US, although rates may be higher in certain geographical areas (DPH 2004; Pfaller and Segreti 2006). Resistance of $P$. aeruginosa to fluoroquinolones, ceftazidime, and imipenem/cilastatin has also increased rapidly; nearly $10 \%$ of $P$. aeruginosa isolates are now resistant to multiple drug classes including cephalosporins, carbapenems, aminoglycosides, and/or fluoroquinolones (Karlowsky et al 2003). Reports of fluoroquinolone resistance are becoming more common among organisms such as E. coli and Proteus spp. that are usually considered to be very susceptible to this class of drugs (Neuhauser et al 2003; Zervos et al 2003).

Anaerobic bacteria such as Bacteroides fragilis, other bacteria of the Bacteroides group, Fusobacterium spp., Prevotella spp., Clostridium spp., and Peptostreptococcus spp. are also relatively common causes of certain cSSTIs. Types of infections in which anaerobes are commonly found include traumatic wounds, infections in diabetic and other immunocompromised patients, and infections associated with ischemic tissue injury (Jones et al 2003). Increasing resistance to common antibiotics such as clindamycin and second-generation cephalosporins (eg, cefoxitin, cefotetan) has been documented among anaerobic bacteria such as $B$. fragilis and organisms of the Bacteroides group (Hecht 2004). However, agents such as metronidazole, piperacillin/ tazobactam, and the carbapenems still maintain excellent activity against most clinically relevant anaerobic bacteria.

This broad range of potential pathogens is further complicated by the fact that many cSSTIs are polymicrobial and represent a variety of mixed infections, ie, presence of aerobic Gram-positive and Gram-negative pathogens as well as anaerobes (Nichols 1999; Fung et al 2003; Stevens et al 
2005). Classic mixed aerobic and anaerobic infections are particularly common in surgical wound infections, necrotizing infections including fasciitis and myositis, complex and perirectal abscesses, diabetic and ischemic ulcers, and complicated cellulitis in diabetics and other immunocompromised patients (Nichols 1999; Pellizer et al 2001; Fung et al 2003; Lipsky et al 2004; Stevens et al 2005; Vinh and Embil 2005).

The recent emergence and spread of antibiotic resistance among both Gram-positive and Gram-negative pathogens have created a significant clinical challenge in the appropriate management of cSSTIs (Colsky et al 1998; Sader et al 2002; Rennie et al 2003). Although a number of drugs for treatment of multidrug-resistant organisms such as MRSA and VRE have become available or are in development (eg, linezolid, daptomycin, tigecycline, dalbavancin), there are few drugs under development for treatment of Gram-negative or anaerobic pathogens which are resistant to the currently available agents. Thus there is a clear need for new antibiotic options for treatment of cSSTI as a result of increasing bacterial resistance. What is equally clear is that the use of currently available agents also needs to be optimized to increase or improve the effective therapeutic options.

\section{Considerations in antibiotic selection}

Appropriate therapeutic strategies for cSSTIs depend on a number of factors including whether the infection is community- versus hospital-acquired in origin, virulence of the infecting pathogens, severity, and presence and type of comorbidities. Surgical intervention will often be required and antibiotic therapy is often considered an adjunctive rather than a primary therapy for certain infections, eg, diabetic foot infections or necrotizing fasciitis (Eron et al 2003; Lipsky et al 2004; Stevens et al 2005). However, adequate antibiotic therapy remains a crucial component of appropriate management of cSSTIs. Empirical antibiotic therapy should usually be promptly initiated after obtaining samples for culture in order to begin appropriate treatment and slow the progression of the infection (Nichols 1999; Eron et al 2003; Stevens et al 2005).

Selection of a specific antibiotic regimen for treatment of cSSTIs depends on many factors such as etiology of the infection (ie, community- or hospital-acquired), knowledge of local or institutional antibiotic susceptibility patterns, availability of suitable antibiotics which provide appropriate spectrum of activity for known or presumed pathogens, pharmacokinetic and pharmacodynamic considerations such as penetration of infected tissues and ability to achieve suitable drug concentrations, adverse effect and drug interaction profiles, and drug cost (Nichols 1999; Eron et al 2003; Fung et al 2003; DiNubile and Lipsky 2004). Because of the broad range of pathogens associated with cSSTIs and the frequency of mixed infections involving both aerobic and anaerobic bacteria, empirical antibiotic regimens are usually broad in spectrum and provide coverage against both Gram-positive and Gram-negative pathogens, with the addition of anaerobic activity also often desired (Nichols 1999; Eron et al 2003; Fung et al 2003; Lipsky et al 2004; Stevens et al 2005). Although streamlining of antibiotic therapy to narrower-spectrum regimens is appropriate once results of microbiological cultures are known, initial antibiotics must be sufficiently broad to cover potential pathogens until other management modalities (ie, surgical intervention) can be instituted.

It has been well documented that increasing resistance among bacterial pathogens results in inappropriate selection of antibiotic therapy for hospital-acquired pneumonia and bloodstream infections (Rello et al 1993; Alvarez-Lerma et al 1996; Luna et al 1997; Leibovici et al 1998; Carmeli et al 1999; Kollef et al 1999; Garnacho-Montero et al 2003). Furthermore, the relationship between inappropriate antibiotic selection and resultant mortality in pneumonia and sepsis has been clearly shown (Rello et al 1993; AlvarezLerma et al 1996; Luna et al 1997; Leibovici et al 1998; Carmeli et al 1999; Kollef et al 1999). Similar data specifically addressing the association between inappropriate antibiotic selection and subsequent adverse outcomes are not as plentiful for cSSTIs. However, one retrospective study of 137 patients with intra-abdominal infections and SSTIs treated with cefotetan, cefoxitin, or ampicillin/sulbactam monotherapy found that isolation of a pathogen resistant to the antibiotic treatment regimen (particularly P. aeruginosa) was an independent risk factor for clinical treatment failure with multivariate analysis (odds ratio $[\mathrm{OR}]=14.9 ; \mathrm{p}=0.001$ ) (Falagas et al 1996). Improper antibiotic selection thus has adverse consequences in cSSTIs as well. Because the bacterial etiology of cSSTIs often involves polymicrobial infections and because of the changing patterns of antibiotic susceptibilities among both Gram-positive and Gram-negative pathogens, the empirical use of antibiotics with a broad spectrum of activity which includes multidrug-resistant organisms is becoming increasingly common and ever more appropriate (Nichols 1999; Eron et al 2003; Fung et al 2003; Lipsky et al 2004; Stevens et al 2005). 


\section{Meropenem in the treatment of cSSTIs}

\section{Antibacterial spectrum of activity}

Meropenem is a broad-spectrum carbapenem antibiotic that possesses excellent activity against both aerobic Grampositive and aerobic Gram-negative bacteria, and also covers common anaerobes. Based on published $\mathrm{MIC}_{90} \mathrm{~s}$ (the minimum inhibitory concentration at which $90 \%$ of tested strains are inhibited), meropenem is generally similar to or slightly less active than imipenem/cilastatin or ertapenem against Gram-positive aerobic bacteria. However, these differences are not likely to be clinically significant for most pathogens because $\mathrm{MIC}_{90}$ s are usually well below recommended susceptibility breakpoints (Wiseman et al 1995; Fish and Singletary 1997; Merrem ${ }^{\circledR}$ product package insert 2005). Meropenem is active against methicillinsusceptible $S$. aureus and most strains of methicillinsusceptible coagulase-negative staphylococci. However, as with other carbapenems, meropenem has poor activity against MRSA and methicillin-resistant coagulase-negative staphylococci. Meropenem also has excellent activity against most streptococci, including Streptococcus pyogenes and $S$. agalactiae. The activity of meropenem against enterococci varies considerably among different species. Most strains of Enterococcus faecalis, E. avium and E. liquefaciens are susceptible or moderately susceptible to meropenem, but most strains of E. faecium and VRE of any species are resistant.

Meropenem has impressive in vitro activity against Gram-negative aerobic bacteria and compares very favorably against both imipenem/cilastatin and ertapenem. Meropenem is generally slightly more active than imipenem/ cilastatin (based on $\mathrm{MIC}_{90} \mathrm{~s}$ ) and similar in activity to ertapenem against clinical isolates of Enterobacteriaceae. Meropenem has shown excellent efficacy against clinical isolates resistant to other $\beta$-lactam agents, including many strains which were highly resistant to third-generation cephalosporins, piperacillin, and aminoglycosides. Meropenem and other carbapenems are considered to be drugs of choice for the treatment of the ESBL-producing Gram-negative bacilli (Paterson et al 2000, 2003). Although meropenem generally has 1- to 2-fold lower $\mathrm{MIC}_{90} \mathrm{~s}$ than imipenem/cilastatin against $P$. aeruginosa, these two drugs appear to be relatively similar in their in vitro activity against clinical isolates of this organism. However, meropenem and imipenem/cilastatin are affected by somewhat different mechanisms of resistance among $P$. aeruginosa strains and cross-resistance among the two drugs is not complete (Fish and Singletary 1997; Rhomberg et al 2005; Quale et al 2006). The Meropenem Yearly Susceptibility Test Information Collection (MYSTIC) surveillance database of antibiotic susceptibilities reports that approximately $7 \%$ of $P$. aeruginosa strains are resistant to imipenem/cilastatin but susceptible to meropenem, while only approximately $1 \%$ of strains were susceptible to imipenem/cilastatin and resistant to meropenem (AstraZeneca 2006). Such discordant susceptibilities are consistent with data published elsewhere (Wiseman et al 1995; Fish and Singletary 1997; Mutnick et al 2004), although the drugs are considered to be equivalent in $P$. aeruginosa activity under most clinical circumstances. Ertapenem is not reliably active against $P$. aeruginosa and is not generally considered to be clinically useful for infections caused by this organism (Fuchs et al 2001; Livermore et al 2001).

Meropenem is also active against most strains of clinically significant anaerobes. Meropenem displayed $\mathrm{MIC}_{90} \mathrm{~s}$ which were quite similar to those for imipenem/ cilastatin and ertapenem in Gram-negative isolates including Bacteroides fragilis, Bacteroides group, and Fusobacterium spp. Meropenem MICs for these organisms are usually substantially lower than those of metronidazole, clindamycin, cefoxitin, or piperacillin/tazobactam. Against Gram-positive anaerobic bacteria such as Clostridium perfringens, C. difficile and Veillonella spp., meropenem is equal to or slightly more active than imipenem/cilastatin, ertapenem, metronidazole, clindamycin, cefoxitin or piperacillin/tazobactam. Meropenem also has excellent activity against clinical isolates of Peptostreptococcus spp., Propionibacterium acnes, Actinomyces spp., and Actinobacillus spp. (Wiseman et al 1995; Fish and Singletary 1997).

\section{Considerations regarding pharmacokinetics/pharmacodynamics and tissue penetration}

Meropenem achieves mean peak plasma concentrations $\left(\mathrm{C}_{\max }\right)$ and minimum plasma concentrations $\left(\mathrm{C}_{\min }\right)$ of approximately $23-25 \mathrm{mg} / \mathrm{L}$ and $0.1-0.2 \mathrm{mg} / \mathrm{L}$, respectively, following the administration of single or multiple 30-minute intravenous infusions of $500 \mathrm{mg}$ to healthy adult volunteers (Maglio et al 2003; Merrem ${ }^{\circledR}$ product package insert 2005). Although $\mathrm{C}_{\min }$ values for meropenem are relatively low, the drug is extremely active against many pathogens commonly found in cSSTIs with $\mathrm{MIC}_{90} \mathrm{~s}$ of $\leq 0.12 \mathrm{mg} / \mathrm{L}$ for most 
Enterobacteriaceae, methicillin-susceptible $S$. aureus and coagulase-negative staphylococci, and streptococci (Rhomberg et al 2005). Studies of carbapenems indicate that the percentage of the dosing interval during which concentrations of free (ie, not protein bound) drug remain above the MIC of the pathogen (referred to as the percent time above MIC, or $\% \mathrm{~T}>\mathrm{MIC}$ ) is the key pharmacodynamic parameter which is related to clinical and microbiological efficacy of the drugs. A $\geq 30 \% \mathrm{~T}>\mathrm{MIC}$ is associated with bacteriostatic activity of the carbapenems, while a $\geq 40 \%$ $\mathrm{T}>\mathrm{MIC}$ is more predictably bactericidal and associated with prevention of the development of resistance (Craig 1998; Turnidge 1998). Meropenem $500 \mathrm{mg}$ every 8 hours achieves $\mathrm{T}>\mathrm{MIC}$ of $\geq 40 \%$ for most susceptible pathogens and would be expected to be effective in the treatment of cSSTIs caused by these organisms at this dose.

Meropenem appears to be widely distributed in various body tissues and fluids (Hutchison et al 1995; Mouton et al 1995; Fish and Singletary 1997). Specifically regarding the use of meropenem in the treatment of cSSTIs, several early studies demonstrated that meropenem achieves concentrations adequate for the treatment of infections caused by susceptible bacteria in skin, burned skin, fascia, skeletal muscle, and blister fluid (Table 2). Meropenem concentrations in these various tissues were quite variable and ranged from $9 \%$ to $85 \%$ of simultaneous serum concentrations; however, the absolute concentrations were nevertheless in excess of the susceptibility breakpoints (MIC $\leq 4 \mathrm{mg} / \mathrm{L}$ ) of bacteria commonly found in cSSTIs (Hutchison et al 1995; Mouton et al 1995; Fish and Singletary 1997). A more recent study also evaluated the pharmacokinetic disposition of meropenem in plasma and cantharidininduced blister fluid (Maglio et al 2003). The mean penetration of meropenem into blister fluid was determined to be $67 \%$ when calculated by comparing the pharmacokinetic area under the concentration-time curve
(AUC) of the drug in blister fluid with the AUC of meropenem in plasma. Additionally, this study found that meropenem doses of $500 \mathrm{mg}$ every 8 hours maintains a $\geq 50 \% \mathrm{~T}>\mathrm{MIC}$ in blister fluid for most susceptible pathogens. This study thus indicated that meropenem $500 \mathrm{mg}$ every 8 hours achieves concentrations in blister fluid sufficient for the treatment of SSTIs caused by susceptible organisms (Maglio et al 2003).

Another recent study used data concerning pharmacokinetics and penetration into blister fluid in order to model the phamacodynamics of meropenem, imipenem/ cilastatin and piperacillin/tazobactam in the treatment of cSSTIs (Ong et al 2005). This study utilized Monte Carlo simulation, a statistical modeling tool which combines both pharmacokinetic and MIC information to predict the probability of achieving desired pharmacodynamic targets with various drug dosing regimens and against specific pathogens. Optimal pharmacodynamic targets with common dosing regimens of each specific agent were defined as $\geq 90 \%$ probability of $\geq 40 \% \mathrm{~T}>\mathrm{MIC}$ (meropenem and imipenem/cilastain) or $\geq 50 \% \mathrm{~T}>\mathrm{MIC}$ (piperacillin/ tazobactam) in serum and blister fluid. Meropenem $500 \mathrm{mg}$ every 8 hours, imipenem/cilastain $500 \mathrm{mg}$ every 8 hours, and piperacillin/tazobactam $3.375 \mathrm{~g}$ every 6 hours were all determined to be optimal choices for empiric treatment of cSSTIs. This study offers further support for the suitability of meropenem as an appropriate agent for the treatment of these infections (Ong et al 2005).

The further application of Monte Carlo simulation to meropenem pharmacodynamics raises important questions related to the optimal dosing of this agent for cSSTIs. Although the previously cited studies provide pharmacokinetic and pharmacodynamic data indicating that meropenem $500 \mathrm{mg}$ every 8 hours is an adequate regimen for infections involving most susceptible pathogens, the adequacy of this regimen specifically for infections

Table 2 Concentration of meropenem in tissues involved in complicated skin and soft tissue infections

\begin{tabular}{|c|c|c|c|c|c|c|}
\hline \multirow[b]{2}{*}{ Body tissue or fluid } & \multirow[b]{2}{*}{$\mathbf{N}$} & \multirow{2}{*}{$\begin{array}{l}\text { Dose } \\
\text { (g) }\end{array}$} & \multirow{2}{*}{$\begin{array}{l}\text { Sample } \\
\text { time (hrs) }\end{array}$} & \multicolumn{2}{|c|}{ Mean drug concentration (mg/L) } & \multirow{2}{*}{$\begin{array}{l}\text { Tissue/fluid:serum } \\
\text { Ratio }\end{array}$} \\
\hline & & & & Tissue/fluid & Serum & \\
\hline Skeletal muscle & 14 & 1 & $0.5-4.5$ & $0.93-6.10$ & - & - \\
\hline Fascia & 18 & 1 & $0.5-5.5$ & $0.99-8.76$ & - & - \\
\hline \multirow[t]{2}{*}{ Skin } & 3 & 0.5 & 0 & 3.97 & 42.6 & $9.6 \%$ \\
\hline & & 0.5 & I & 3.4 & 11.9 & $33.5 \%$ \\
\hline \multirow[t]{2}{*}{ Burned skin } & 2 & 1 & 0 & 5.5 & 64.3 & $8.8 \%$ \\
\hline & & I & I & 9.2 & 14.8 & $61.6 \%$ \\
\hline Blister fluid & 8 & $10 \mathrm{mg} / \mathrm{kg}$ & $3.5-4.5$ & 1.36 & 1.44 & $85 \%$ \\
\hline Blister fluid & 6 & 1 & $3.5-4.5$ & 4.94 & 2.07 & $>38 \%$ \\
\hline
\end{tabular}


involving $P$. aeruginosa is less clear. Other published Monte Carlo evaluations of meropenem pharmacodynamics indicate that even regimens of $1 \mathrm{~g}$ every 8 hours often fail to produce desired ( $\geq 90 \%$ probability), and sometimes not even acceptable ( $\geq 80 \%$ probability) likelihood of attaining the desired pharmacodynamic target of $\geq 40 \% \mathrm{~T}>\mathrm{MIC}$ for P. aeruginosa (Kuti et al 2004; Masterton et al 2005). While the probabilities of achieving specified pharmacodynamic targets for $P$. aeruginosa isolates from different geographic regions ranged from $63 \%$ to $91 \%$ with meropenem $1 \mathrm{~g}$ every 8 hours, probabilities of target attainment with the regimen of $500 \mathrm{mg}$ every 8 hours ranged from only $59 \%$ to $81 \%$ (Kuti et al 2004; Masterton et al 2005). These analyses were performed utilizing MIC data from the MYSTIC database, but $P$. aeruginosa susceptibilities of isolates obtained from different geographic regions, specific institutions, and even individual patients may be very different from those in MYSTIC and would therefore yield quite different probabilities of pharmacodynamic target attainment (either higher or lower). Although a previous analysis of meropenem for cSSTIs indicated a high probability of achieving desired pharmacodynamic goals with the regimen of $500 \mathrm{mg}$ every 8 hours, this analysis was based on pooled susceptibility data in which MIC data were weighted according to the prevalence of causative pathogens encountered in cSSTIs and did not report probability of target attainment specifically for $P$. aeruginosa (Ong et al 2005) Based on pharmacodynamic considerations, it appears that meropenem $500 \mathrm{mg}$ every 8 hours is not necessarily optimal for the treatment of cSSTIs or other infections when P. aeruginosa is suspected or documented. Meropenem $1 \mathrm{~g}$ every 8 hours may be a more preferred regimen in these cases.

The elimination half-life of meropenem is approximately one hour in patients with normal renal function. The major route of meropenem elimination is urinary excretion of unchanged drug, with renal clearance $\left(\mathrm{CL}_{\mathrm{R}}\right)$ accounting for $58 \%-83 \%$ of total systemic clearance of the drug and up to $98 \%$ of each dose eventually excreted in the urine as unchanged meropenem and as a pharmacologically inactive, open $\beta$-lactam metabolite (Wiseman et al 1995; Fish and Singletary 1997). It appears that meropenem pharmacokinetics in children $\geq 6$ months of age are very similar to those seen in adults.

Because many cSSTIs occur in the setting of diabetes mellitus and other acute or chronic illnesses associated with renal dysfunction, the disposition of meropenem in patients with renal impairment is of relevance. Following intravenous administration, meropenem $\mathrm{AUC}, \mathrm{CL}_{\mathrm{R}}$, and total systemic clearance are altered in proportion to the degree of renal impairment present. Dosage adjustments are therefore required in patients with moderate to severe renal insufficiency and calculated creatinine clearances of $<50 \mathrm{~mL} /$ minute in order to prevent excessive drug accumulation. Dosage adjustments of meropenem in elderly patients are also based on renal function with no other apparent age-related pharmacokinetic alterations. The pharmacokinetics of meropenem are not significantly altered in patients with hepatic impairment and no dosage adjustments are required (Wiseman et al 1995; Fish and Singletary 1997).

\section{Clinical data}

At least three studies have evaluated meropenem in the treatment of cSSTIs (Table 3) (Lami et al 1991; Nichols et al 1995; Fabian et al 2005). The first of these was a small prospective, open-label study comparing meropenem with imipenem/cilastatin in patients with soft tissue infections requiring hospitalization, surgical drainage, and/or debridement in addition to antibiotic therapy (Lami et al 1991). Pertinent among the exclusion criteria were patients with neutropenia or diabetic foot infections requiring amputation; clinically evaluable patients were required to have documented bacterial pathogens. Patients were randomized to receive either meropenem $500 \mathrm{mg}$ every 8 hours ( 23 evaluable patients) or imipenem/cilastatin $500 \mathrm{mg}$ every 6 hours (21 patients); patients in both groups were treated for a mean duration of approximately 7 days. Patients in the two treatment groups were similar with regards to demographics, severity and type of infections. S. aureus, streptococci, and E. coli were the most common pathogens with a mean of three bacterial isolates per patient. Satisfactory clinical responses were demonstrated in $100 \%$ of patients in both treatment groups; microbiological response rates and the number of infections caused specifically by $P$. aeruginosa were not reported. Both antibiotic regimens were well tolerated with an apparently low reported incidence of adverse events.

A second study also evaluated the efficacy of meropenem compared with imipenem/cilastatin in the treatment of severe infections (Nichols et al 1995). This was a prospective, randomized, multicenter, open-label study. The primary inclusion criteria were requirement for hospitalization and treatment with parenteral antibiotics; the specific types of infections eligible for inclusion in the study were not well defined. Relevant exclusion criteria included neutropenia, 
Table 3 Summary of meropenem clinical efficacy trials in complicated skin and soft tissue infections

\begin{tabular}{|c|c|c|c|c|c|c|c|}
\hline \multirow[b]{2}{*}{ Reference } & \multirow[b]{2}{*}{ Study type } & \multirow[b]{2}{*}{$\begin{array}{l}\text { Evaluable } \\
\text { patients } \\
(\mathrm{N})\end{array}$} & \multirow[b]{2}{*}{ Regimen } & \multirow[b]{2}{*}{$\begin{array}{l}\text { Mean } \\
\text { duration of } \\
\text { therapy } \\
\text { (days) }\end{array}$} & \multicolumn{2}{|c|}{ Response } & \multirow[b]{2}{*}{$\begin{array}{l}\text { Drug- } \\
\text { related } \\
\text { AE (\%) }\end{array}$} \\
\hline & & & & & $\begin{array}{l}\text { Clinical } \\
(\%)\end{array}$ & $\begin{array}{l}\text { Bacteriologic } \\
(\%)\end{array}$ & \\
\hline \multirow[t]{2}{*}{ Lami et al I99I } & $P, R, N B$ & 23 & M 500 mg q8h & 6.4 & 100 & NR & NR \\
\hline & & 21 & $\mathrm{l} / \mathrm{C} 500 \mathrm{mg}$ q6h & 6.5 & 100 & NR & NR \\
\hline \multirow{2}{*}{ Nichols et al 1995} & $\mathrm{P}, \mathrm{R}, \mathrm{MC}, \mathrm{NB}$ & 123 & M 500 mg q8h & 7.1 & 98 & 94 & 14 \\
\hline & & 126 & I/C $500 \mathrm{mg} \mathrm{q6h}$ & 7.3 & 95 & 91 & 16 \\
\hline \multirow[t]{2}{*}{ Fabian et al 2005} & $\mathrm{P}, \mathrm{R}, \mathrm{MC}, \mathrm{DB}$ & 261 & M 500 mg q8h & $5.8^{\mathrm{a}}$ & 86.2 & 88.5 & 9.0 \\
\hline & & 287 & I/C $500 \mathrm{mg} q 8 \mathrm{~h}$ & $6.0^{\mathrm{a}}$ & 82.9 & 83.1 & 10.8 \\
\hline
\end{tabular}

Note: ${ }^{2}$ Numbers in table represent duration of parenteral therapy only. Approximately $50 \%$ of patients in each treatment group were switched to oral therapy and treated for an additional mean of 9.3 and 9.0 days in meropenem and $\mathrm{I} / \mathrm{C}$ groups, respectively.

Abbreviations: AE, adverse events; DB, double-blind; I/C, imipenem/cilastatin; M, meropenem; MC, multicentered; NB, non-blinded; NR, not reported; P, prospective; $\mathrm{R}$, randomized.

rapidly progressive disease, and severe peripheral vascular disease likely to require amputation. Patients received either meropenem $500 \mathrm{mg}$ every 8 hours (123 evaluable patients) or imipenem/cilastatin $500 \mathrm{mg}$ every 6 hours (126 patients); patients in both groups were treated for a mean duration of approximately 6 days. Primary study endpoints were clinical and bacteriologic responses at the end of therapy and at a follow-up visit 2 to 4 weeks after the end of therapy. Treatment groups were similar with respect to patient demographics, diagnoses, or required surgical procedures during treatment. Greater than $97 \%$ of infections were community-acquired and included patients with diabetes, peripheral vascular disease, and injection drug use-related infections. Approximately half of all infections were monomicrobial and involved a Gram-positive organism in $86 \%$ of these patients. Polymicrobic infections were documented in $48 \%$ of meropenem-treated patients and $45 \%$ of patients receiving imipenem/cilastatin. The overall distribution of pathogens in these patients was $58 \%$ aerobic Gram-positive bacteria, 22\% aerobic Gram-negatives, and $20 \%$ anaerobes. Satisfactory clinical responses at end of treatment were similar between the groups treated with meropenem and imipenem/cilastatin (98\% versus $95 \%$, respectively; $95 \%$ confidence interval [CI], -2.29 to 6.93 ). Microbiological response rates at end of treatment in the meropenem and imipenem/cilastatin groups were also similar ( $94 \%$ and $91 \%$, respectively; $95 \% \mathrm{CI},-2.73$ to 10.39); however, meropenem was statistically better than imipenem/cilastatin in overall eradication of pathogens $(96 \%$ vs $90 \%$, respectively; $\mathrm{p}=0.007$ ) and eradication of grampositive aerobes ( $96 \%$ vs $88 \%$, respectively; $\mathrm{p}=0.01)$. Although P. aeruginosa was reported to be eradicated in 9 of 11 patients $(82 \%)$, this rate was somewhat lower than that reported for other Gram-negative pathogens (48 of 50, 96\%). Clinical efficacy rates in meropenem and imipenem/ cilastatin groups at the 2- to 4-week follow-up visit were $92 \%$ and $89 \%$, respectively ( $\mathrm{p}=0.60$ ), while microbiological efficacy at follow-up was $92 \%$ versus $82 \%$, respectively $(\mathrm{p}=0.11)$. Superinfection due to $S$. aureus was documented in one meropenem-treated patient; the incidence of other drug-related adverse events was similar between groups.

Although these first two studies provide support for meropenem's efficacy in the treatment of cSSTIs, they had potential limitations in assessing the role of meropenem specifically for complicated infections. First, it is not clear whether these studies actually evaluated the treatment of complicated infections as these are currently defined. Both studies included patients with infections requiring hospitalization and surgical intervention, so these infections do appear to have been more severe or complicated in nature, or both. However, the sites and types of infections eligible for inclusion in the studies were not always well defined. The $100 \%$ efficacy rates reported in the first study are also somewhat suspect for complicated infections (Lami et al 1991); this may reflect the unblinded nature of the study rather than the possibility that the infections were not truly complicated in nature. In the second study (Nichols et al 1995), the infections were overwhelmingly communityacquired in origin. Although these were appropriate for evaluation, the study did not represent the full spectrum of infections (eg, surgical wound infections, other nosocomial infections) of importance in cSSTIs. Thus although the overall results of these two studies were favorable for meropenem, they both had relative limitations regarding study design and/or enrolled patient populations which make 
the significance and applicability of the results difficult to fully evaluate.

The best and most recent study of meropenem in the treatment of cSSTIs overcame the deficiencies of the two earlier, previously-described studies. This large prospective, randomized, multicenter, international, double-blind study compared the efficacy and safety of meropenem $500 \mathrm{mg}$ every 8 hours versus imipenem/cilastatin $500 \mathrm{mg}$ every 8 hours in hospitalized patients with cSSTIs (Fabian et al 2005). Infections classified as complicated for purposes of this study included the following: complicated cellulitis (ie, in the setting of diabetes, involving the perineum, or deep tissue involvement evidenced by necrosis, tissue fluctuance, bacteremia, or need for surgical incision); surgical site infections; traumatic infections; infected diabetic and ischemic ulcers; complex or perirectal abscesses, or other bacterial infections requiring hospitalization, surgical intervention, and parenteral antibiotics. In addition, all patients were required to have had specimens for culture and susceptibility testing obtained before study enrollment; any pathogens known to be present at study enrollment were required to be susceptible to study drugs. Pertinent exclusion criteria included neutropenia, underlying osteomyelitis, severe peripheral vascular disease likely to require amputation, necrotizing fasciitis, pressure ulcerations, or infected prosthetic materials. Concomitant therapy with vancomycin or other systemic antibiotics was not permitted; patients could be switched to oral antibiotics of the investigators' choice after a minimum of three days of parenteral therapy. The primary study endpoints were clinical efficacy (cure versus failure or relapse) in clinically evaluable (CE) and modified intent-to-treat (MITT, patients meeting all eligibility criteria and receiving $\geq 1$ dose of drug) populations at a follow-up test-of-cure (TOC) visit 7 to 28 days after completion of all antibiotics. Secondary endpoints included clinical efficacy in the intent-to-treat (ITT, all patients receiving $\geq 1$ dose of drug whether or not they met eligibility criteria) population at the TOC follow-up visit, as well as clinical efficacy in the ITT, MITT, and CE populations at the end-of-therapy visit occurring within 24 hours of completion of antibiotic therapy.

A total of 1076 patients were enrolled into the study. Of these, final study populations treated with meropenem and imipenem/cilastatin included 510 and 527 patients, respectively, in the ITT groups; 334 and 358 patients, respectively, in the MITT groups; and 261 and 287 patients, respectively, in the $\mathrm{CE}$ groups. Treatment groups were similar in respect to patient demographics, types of infection, and underlying medical conditions. Approximately $93 \%$ of patients in each group had infections classified as moderate or severe in nature, and $80 \%$ in each treatment arm had deep tissue involvement. Additionally, $68 \%$ of patients treated with meropenem and $70 \%$ of those receiving imipenem/cilastatin required surgical intervention. In contrast to the two earlier studies, approximately $18 \%$ of patients in both groups had surgical site or traumatic wound infections. Isolated pathogens were also evenly represented among the two treatment groups. Aerobic Gram-positive organisms accounted for $57 \%$ of pre-treatment pathogens, while aerobic Gram-negative pathogens were found in $26 \%$ of patients and anaerobes in 17\% (Table 4). Polymicrobial infections were microbiologically documented in $38 \%$ of patients at pre-treatment baseline.

The mean duration of therapy with intravenous therapy was approximately 6 days in both groups, similar numbers of patients in each group were switched to oral antibiotics (approximately $50 \%$ ), and the mean duration of oral antibiotics was also similar between groups (approximately 9 days). Evaluation of the primary study endpoints demonstrated equivalent efficacy between meropenem and imipenem/cilastatin within both the CE $(86.2 \%$ vs $82.9 \%$, respectively; $95 \% \mathrm{CI},-2.8$ to 9.3 ) and MITT populations ( $73.1 \%$ vs $74.9 \%$, respectively; $95 \%$ CI, -8.4 to 4.7 ). There were also no statistically significant differences between meropenem and imipenem/cilastatin among the secondary endpoints. Clinical efficacy rates in the meropenem and imipenem/cilastatin groups within the ITT population at the

Table 4 Pre-treatment pathogens isolated from patients with complicated skin and soft tissue infections (Fabian et al 2005)

\begin{tabular}{lll}
\hline Total pathogens & $\mathbf{1 4 4 4}$ & $\mathbf{( 1 0 0 \% )}$ \\
\hline Gram-positive aerobes & $\mathbf{8 2 7}$ & $\mathbf{( 5 7 \% )}$ \\
Staphylococcus aureus (MS) & 295 & $(20 \%)$ \\
Staphylococcus aureus (MR) & 118 & $(8 \%)$ \\
Streptococcus pyogenes & 89 & $(6 \%)$ \\
Streptococcus agalactiae & 59 & $(4 \%)$ \\
Enterococcus faecalis & 52 & $(4 \%)$ \\
Gram-negative aerobes & $\mathbf{3 7 8}$ & $(\mathbf{2 6 \% )}$ \\
Escherichia coli & 64 & $(4 \%)$ \\
Pseudomonas aeruginosa & 53 & $(4 \%)$ \\
Proteus spp. & 52 & $(4 \%)$ \\
Klebsiella spp. & 41 & $(3 \%)$ \\
Anaerobes & $\mathbf{2 3 9}$ & $\mathbf{( 1 7 \% )}$ \\
Bacteroides fragilis & 74 & $(5 \%)$ \\
Peptostreptococcus spp. & 63 & $(4 \%)$ \\
Prevotella spp. & 46 & $(3 \%)$ \\
Monomicrobial infections & $\mathbf{8 9 5}$ & $(\mathbf{6 2 \% )}$ \\
Polymicrobial infections & $\mathbf{5 4 9}$ & $\mathbf{( 3 8 \% )}$ \\
\hline
\end{tabular}

Abbreviations: MS, methicillin-susceptible; MR, methicillin-resistant. 
TOC visit (approximately 57\% vs 61\%, respectively; 95\% CI, -9.8 to 2.1$)$, and within the CE (93.5\% vs $92.3 \%$, respectively; approximate $95 \% \mathrm{CI},-3.1$ to 5.4$)$, MITT (91.0\% vs 91.1\%, respectively; approximate 95\% CI, -4.3 to 4.2$)$, and ITT ( $81.0 \%$ vs $83.5 \%$, respectively; approximate $95 \% \mathrm{CI},-7.1$ to 2.0 ) populations at the end-of-treatment evaluations were all similar among the two treatment groups. Again, no significant differences in efficacy among the meropenem and imipenem/cilastatin groups were observed when patients were divided into subgroups according to demographic parameters, types of infection, or infecting pathogens. Clinical cure rates specifically in patients infected with $P$. aeruginosa and treated with meropenem and imipenem/cilastatin were $73.3 \%(11 / 15)$ and $86.7 \%(13 /$ $15)$, respectively $(\mathrm{p}=0.652)$. The incidence of drug-related adverse events was similar between meropenem and imipenem/cilastatin groups ( $9.0 \%$ vs $10.8 \%$, respectively).

Additional data from this study regarding the bacteriology and clinical efficacy of meropenem and imipenem/cilastatin specifically in diabetic patients were recently presented at an international meeting (Embil et al 2005). A total of 398 patients (38\%) enrolled into the study had diabetes mellitus. Of these patients, 254 (82\%) had infections involving the leg or foot, or both, most commonly complicated cellulitis, complex abscess, or infected ulcers. The distribution of pathogens in these patients was $58 \%$ aerobic Gram-positive bacteria, 27\% aerobic Gramnegatives, and 15\% anaerobes. This distribution of pathogens was similar to that seen in non-diabetic patients, although the frequency of polymicrobial infection was greater in diabetic versus non-diabetic patients ( $44 \%$ versus $34 \%$, respectively; $p=0.002$ ). Clinical efficacy rates among all enrolled patients at follow-up were significantly higher with meropenem vs. imipenem/cilastatin in diabetic patients ( $86 \%$ vs $72 \%$, respectively; $\mathrm{p}<0.0001$ ) but not in nondiabetic patients ( $87 \%$ vs $89 \%$, respectively; $\mathrm{p}=0.322$ ). Clinical efficacy rates among microbiologically evaluable diabetic patients were also significantly higher for infections caused by various bacteria among meropenem-treated patients compared with those receiving imipenem/cilastatin (Gram-positive aerobes, 87\% vs 70\%; Gram-negative aerobes, $74 \%$ vs $60 \%$; anaerobes, $94 \%$ vs $71 \%$; and polymicrobial infections, $88 \%$ vs $65 \%$, respectively; $\mathrm{p}<0.0001$ for each of the four comparisons). Possible reasons for these reported differences in efficacy are not clear. Although previous studies used imipenem/cilastatin doses of $500 \mathrm{mg}$ every 6 hours (Lami et al 1991; Nichols et al 1995), the lower dose of $500 \mathrm{mg}$ every 8 hours used in this third study has been shown to be apparently adequate for the treatment of cSSTIs based on pharmacodynamic modeling (Ong et al 2005). In addition, since this abstract reported data from an unplanned subgroup analysis of the original study, the post-hoc statistical comparisons do not necessarily indicate that meropenem is superior to imipenem/cilastatin in the treatment of cSSTIs in diabetic patients. However, the data do indicate that meropenem is at least comparable with imipenem/cilastatin and is quite an effective agent in the treatment of such infections.

Based on available data as reviewed in this article, meropenem was approved by the FDA on May 25, 2005 for the treatment of cSSTIs at a dose of $500 \mathrm{mg}$ intravenously every 8 hours. Meropenem is specifically indicated for cSSTIs caused by the following pathogens: S. aureus (methicillin susceptible only), S. pyogenes, S. agalactiae, viridans group streptococci, E. faecalis (vancomycin susceptible only), E. coli, P. mirabilis, P. aeruginosa, $B$. fragilis, or Peptostreptococcus spp.

\section{Role of meropenem in cSSTIs}

Complicated skin and soft tissue infections continue to represent a considerable clinical challenge. The underlying conditions such as diabetes mellitus, peripheral vascular disease, surgery, and immunosuppression that predispose to cSSTIs also make them potentially less responsive to traditional antibiotic therapy, thus necessitating hospital admission for parenteral antibiotics and surgical interventions. The broad range of bacterial pathogens likely to be encountered in cSSTIs, together with the changing epidemiology of susceptibilities to currently available antibiotics, adds to the complexity of managing these infections. As previously discussed, meropenem has excellent activity against most bacteria commonly associated with cSSTIs, often including $P$. aeruginosa and ESBLproducing organisms which are resistant to other $\beta$-lactam antibiotics including third-generation cephalosporins. The status of carbapenems as the drugs of choice in the treatment of infection due to ESBL-producing organisms makes meropenem and other agents of this class particularly attractive in geographic areas with high prevalence of ESBL producers. Meropenem's broad antimicrobial spectrum of action is thus well suited to either empiric treatment of cSSTIs when specific pathogens are unknown, as well as definitive treatment of mixed bacterial infections and/or those caused by multidrug-resistant organisms once specific pathogens and susceptibilities have been documented. However, because of the recent emergence of community- 
acquired MRSA as an important pathogen, the addition of vancomycin, linezolid, or some other agent with good activity against MRSA should be considered for communityacquired infections in which $S$. aureus is a suspected pathogen.

The use of broad-spectrum antibiotics for the initial treatment of cSSTIs has been addressed in recent practice guidelines from the Infectious Diseases Society of America (Lipsky et al 2004; Stevens et al 2005). Broad-spectrum agents which provide good activity against Gram-positive aerobes, Gram-negative aerobes, and anaerobes have been recommended as appropriate initial choices for a variety of cSSTIs including complicated cellulitis, severe diabetic foot infections, surgical wound infections, infections in immunocompromised hosts, severe animal or human bite wounds, and necrotizing infections (Lipsky et al 2004; Stevens et al 2005). There are currently a number of agents that could potentially be appropriate for use in these types of infections. $\beta$-Lactam $/ \beta$-lactamase inhibitor combinations such as piperacillin/tazobactam have been extensively used for these indications due to their favorable spectrum of activity and proven efficacy in a wide variety of infection types (Tan et al 1993; Tassler et al 1993; Lipsky et al 2004, 2005). The new glycylcycline-class drug, tigecycline, has also been shown to have good efficacy in the treatment of cSSTIs and was approved in the US for this indication in June 2005 (Breedt et al 2005; Wilcox 2005). Carbapenems such as imipenem/cilastatin and, more recently, ertapenem have also been shown to be very effective in the initial treatment of severe cSSTIs (Grayson et al 1994; Graham et al 2002; Lipsky et al 2005). Although the carbapenems are too broad in spectrum for routine first-line use in all types of infections, they are particularly well suited and recommended as appropriate agents for infections likely to involve mixed and/or multidrug-resistant Gram-negative pathogens such as surgical wound and other hospitalacquired infections, severe diabetic foot infections, and infections in immunocompromised patients (Fung et al 2003; DiNubile and Lipsky 2004; Lipsky et al 2004; Stevens et al 2005).

Meropenem has shown excellent efficacy in controlled clinical studies in a variety of types of moderate to severe infections in seriously ill patients (Wiseman et al 1995; Fish and Singletary 1997). Clinical studies directly comparing meropenem versus imipenem/cilastatin in the treatment of intra-abdominal, lower respiratory tract, SSTIs, urinary tract infections, and exacerbations of chronic obstructive pulmonary disease (COPD) generally found these two agents to be comparable in clinical and microbiological efficacy (Colardyn and Faulkner 1996; Garau et al 1997). As discussed in this article, at least three clinical studies have also shown meropenem to be comparable with imipenem/cilastatin in the treatment of cSSTIs, one of these being among the largest and best-designed studies of cSSTIs ever performed (Fabian et al 2005). While meropenem and the other carbapenems are not generally recommended for initial treatment of all cSSTIs because their extremely broad spectrum of activity is not necessarily required and excessive use of these agents is undesirable, their use is appropriate and recommended for initial treatment in certain selected patients as previously discussed. Meropenem is an appropriate antibiotic choice in those patients in whom use of a carbapenem is recommended for infections involving pathogens known to be resistant to other narrower-spectrum, more preferred agents.

Although meropenem is indicated by the US FDA for treatment of cSSTIs caused by $P$. aeruginosa, the approved regimen of $500 \mathrm{mg}$ every 8 hours may not necessarily be the preferred regimen for empiric treatment of cSSTIs in higher-risk patients in which $P$. aeruginosa is more likely to occur, ie, hospital-acquired infections, severe diabetic foot infections, and infections in immunocompromised patients. As previously discussed, pharmacokinetic/pharmacodynamic analyses indicate that this regimen may not be as favorable as higher doses, ie, $1 \mathrm{~g}$ every 8 hours, in reliably achieving desired pharmacodynamic targets for treatment of $P$. aeruginosa infections (Kuti et al 2004; Masterton et al 2005). Additionally, although clinical studies of meropenem in the treatment of cSSTIs were overall very favorable, relatively few $P$. aeruginosa were isolated in these studies and the overall response rate in these patients $(20 / 26,77 \%)$ was somewhat lower than the overall clinical and microbiological efficacy rates reported in these three studies ( $86 \%$ to $100 \%$ and $88 \%$ to $94 \%$, respectively) (Lami et al 1991; Nichols et al 1995; Fabian et al 2005). These findings, together with considerations regarding potential alterations in drug pharmacokinetics and tissue penetration, increasing $P$. aeruginosa resistance, and potential differences in local and institutional susceptibility patterns, strongly suggest that the use of higher daily doses of meropenem may be more prudent when $P$. aeruginosa is suspected or until specific susceptibilities can be determined in documented strains

In addition to meropenem's favorable spectrum of activity and proven clinical efficacy, meropenem is also well tolerated and has few serious toxicities associated with its 
use (Wiseman et al 1995; Fish and Singletary 1997; Norrby and Gildon 1999). Although carbapenems have been associated with seizures and other central nervous system (CNS) toxicities, the rate of meropenem-associated CNS toxicity appears to be less than that of imipenem/cilastatin and probably similar to ertapenem (Wiseman et al 1995; Fish and Singletary 1997; Norrby and Gildon 1999; Teppler et al 2004). The incidence of seizures during meropenem therapy is very low and appears to be $0.05 \%$ to $0.08 \%$ (Fish and Singletary 1997; Norrby and Gildon 1999). This potential difference in CNS toxicities between meropenem and imipenem/cilastatin may be most relevant in higherrisk patients such as those with head trauma, seizure disorders, or other underlying CNS pathology. The overall safety and tolerability of meropenem appears to be similar to other carbapenems (Fish and Singletary 1997; Norrby and Gildon 1999).

The average wholesale price (AWP) of meropenem and other very broad-spectrum agents which are currently used as monotherapy for the treatment of cSSTIs are shown in Table 5 (Red Book 2005). Prices shown are based on a 7 day course of therapy at FDA-approved doses of the respective drugs in patients with good renal function. Although meropenem is most easily compared against other carbapenems, piperacillin/tazobactam and tigecycline are also appropriate for treatment of certain cSSTIs and could potentially be used as alternatives to carbapenems in many patients (Fung et al 2003; Lipsky et al 2004; Breedt et al 2005; Stevens et al 2005; Wilcox 2005). Although ertapenem and tigecycline have poor activity against $P$. aeruginos $a$ and are not strictly interchangeable with meropenem for all infections, ertapenem does offer potential cost advantages in the treatment of those infections for which it is appropriate (eg, when $P$. aeruginosa is not a likely pathogen). Compared with imipenem/cilastatin, a drug which is quite similar to meropenem in terms of activity and clinical efficacy, meropenem may in fact be more cost-effective. However, actual costs at individual institutions will vary considerably according to specific contract pricing agreements and costs within countries outside of the US. The selection of one agent over another may very well depend on prices available to the specific institution. Local antibiograms are also important to consider in the selection of a specific agent.

Meropenem possesses many favorable qualities and some possible advantages relative to other agents which are commonly used in the treatment of cSSTIs. These advantages may include good activity against pathogens (particularly Gram-negative aerobes) with increasing resistance to cephalosporins and extended-spectrum penicillins; the ability to effectively treat many cSSTIs with a single-drug regimen rather than a combination of agents in order to provide adequate coverage of suspected or documented pathogens; incomplete cross-resistance with imipenem/cilastatin and slightly greater activity against $P$. aeruginosa; potentially decreased CNS toxicity compared with imipenem/cilastatin; and potential cost advantages. Conversely, potential disadvantages of meropenem use include an excessively broad spectrum of activity for treatment of many cSSTIs in which $P$. aeruginosa, anaerobes, and/or multidrug-resistant pathogens are not likely to be present; a broad spectrum of activity which may increase selective pressure for development of resistance among organisms such as $P$. aeruginosa; the need for addition of another agent when MRSA is possibly present in community-acquired infections; the need for three-timesdaily dosing; and potentially higher costs relative to some alternative agents. Based on available data, meropenem should be considered relatively interchangeable with imipenem/cilastatin for treatment of cSSTIs and would be preferred over ertapenem for certain patients who are at high risk of infection with $P$. aeruginosa. Meropenem is an

Table 5 Approximate treatment costs for a 7-day course of therapy with selected agents in the treatment of complicated skin and soft tissue infection

\begin{tabular}{|c|c|c|c|}
\hline Drug & US trade name and manufacturer & Dosing regimen & Cost \\
\hline \multicolumn{4}{|l|}{ Carbapenems } \\
\hline Meropenem & Merrem $^{\circledR}$; AstraZeneca, Wilmington, Delaware, USA & $500 \mathrm{mg}$ every 8 hours & $\$ 630$ \\
\hline Imipenem/cilastatin & Primaxin $^{\circledR} ;$ Merck \& Co., Whitehouse Station, New Jersey, USA & $500 \mathrm{mg}$ every 8 hours & $\$ 722$ \\
\hline Ertapenem & Invanz ${ }^{\circledR}$; Merck \& Co., Whitehouse Station, New Jersey, USA & I g every 24 hours & $\$ 360$ \\
\hline \multicolumn{4}{|l|}{ Other agents } \\
\hline Piperacillin/tazobactam & Zosyn $^{\circledR}$;Wyeth Pharmaceuticals, Philadelphia, Pennsylvania, USA & $3.375 \mathrm{~g}$ every 6 hours & $\$ 482$ \\
\hline Tigecycline & Tygacil $^{\mathrm{TM}}$;Wyeth Pharmaceuticals, Philadelphia, Pennsylvania, USA & $50 \mathrm{mg}$ every 12 hours $^{\text {a }}$ & $\$ 735$ \\
\hline
\end{tabular}

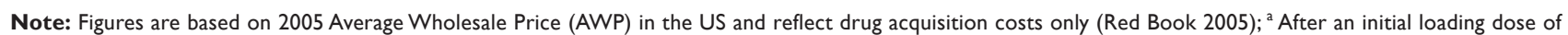
$100 \mathrm{mg}$. 
effective and safe agent for treatment of cSSTIs for which carbapenems are deemed appropriate.

\section{Summary}

Meropenem is a broad-spectrum carbapenem antibiotic with excellent activity against many aerobic Gram-positive, aerobic Gram-negative, and anaerobic organisms commonly associated with cSSTIs. At least three clinical studies, including one of the largest prospective, randomized, double-blind studies of cSSTIs yet conducted, have shown that meropenem is comparable in clinical efficacy with imipenem/cilastatin and is well tolerated in the treatment of these infections. Carbapenems are currently recommended as appropriate agents in the initial treatment of certain types of cSSTIs including those likely to involve mixed and/or multidrug-resistant pathogens, ie, surgical wound and other hospital-acquired infections, severe diabetic foot infections, and infections in immunocompromised patients. When used for appropriate types of cSSTIs in carefully selected patients, and with the addition of a second agent for coverage of MRSA when appropriate, meropenem is an effective and viable alternative for monotherapy of these challenging infections. The regimen of meropenem $500 \mathrm{mg}$ every 8 hours is appropriate for the treatment of infections caused by most pathogens. However, this author recommends the use of higher doses (ie, $1 \mathrm{~g}$ every 8 hours) for empiric treatment of patients at higher risk of infection with $P$. aeruginosa or when this specific pathogen is actually documented to be present.

\section{References}

Alvarez-Lerma F; ICU-Acquired Pneumonia Study Group. 1996. Modification of empiric antibiotic treatment in patients with pneumonia acquired in the intensive care unit. Intensive Care Med, 22:387-94.

AstraZeneca Pharmaceuticals. 2006. MYSTIC surveillance study online database [online]. Accessed on 21 April 2006. URL: http:// www.mystic-data.org/main.htm.

Bowler PG, Duerden BI, Armstrong DG. 2001. Wound microbiology and associated approaches to wound management. Clin Microbiol Rev, 14:244-69.

Breedt J, Teras J, Gardovskis J, et al. 2005. Safety and efficacy of tigecycline in treatment of skin and skin structure infections: results of a double-blind phase 3 comparison study with vancomycinaztreonam. Antimicrob Agents Chemother, 49:4658-66.

Bukharie H, Abdelhadi M, Saeed I, et al. 2001. Emergence of methicillinresistant Staphylococcus aureus as a community pathogen. Diagn Microbiol Infect Dis, 40:1-4.

Bush K. 2001. New -lactamases in gram-negative bacteria: diversity and impact on the selection of antimicrobial therapy. Clin Infect Dis, 32:1085-9.

Carmeli Y, Troillet N, Karchmer AW, et al. 1999. Health and economic outcomes of antibiotic resistance in Pseudomonas aeruginosa. Arch Intern Med, 159:1127-32
[CDCP] Centers for Disease Control and Prevention. 2001. Incidence of soft tissue infections: San Francisco General Hospital - 1996-2000. Morbid Mortal Weekly Report, 50:381-4.

Chow JW, Satishchandran V, Snyder TA, et al. 2006. In vitro susceptibilities of aerobic and facultative gram-negative bacilli isolated from patients with intra-abdominal infections worldwide: the 2002 Study for Monitoring Antimicrobial Resistance Trends (SMART). Surg Infect, 6:439-47.

Colardyn F, Faulkner KL. 1996. Intravenous meropenem versus imipenem/ cilastatin in the treatment of serious bacterial infections in hospitalized patients. Meropenem Serious Infection Study Group. J Antimicrob Chemother, 38:523-37.

Colsky AS, Kirsner RS, Kerdel FA. 1998. Analysis of antibiotic susceptibilities of skin wound flora in hospitalized dermatology patients. The crisis of antibiotic resistance has come to the surface. Arch Dermatol, 134:1006-9.

Craig WA. 1998. Pharmacokinetic/pharmacodynamic parameters: rationale for antibacterial dosing of mice and men. Clin Infect Dis, 26:1-10.

DiNubile MJ, Lipsky BA. 2004. Complicated infections of skin and skin structures: when the infection is more than skin deep. J Antimicrob Chemother, 53(Suppl S2):ii37-ii50.

Doern GV, Jones RN, Pfaller MA, et al. 1999. Bacterial pathogens isolated from patients with skin and soft tissue infections: frequency of occurrence and antimicrobial susceptibility patterns from the SENTRY Antimicrobial Surveillance Program. Diagn Microbiol Infect Dis, 34:65-72.

[DPH] US Department of Public Health and Human Services, Public Health Service. 2004. National Nosocomial Infections Surveillance (NNIS) System Report, data summary from January 1992 through June 2004, issued October 2004. Am J Infect Control, 32:470-85.

Eady EA, Cove JH. 2003. Staphylococcal resistance revisited: communityacquired methicillin resistant Staphylococcus aureus - an emerging problem for the management of skin and soft tissue infections. Curr Opin Infect Dis, 16:103-24.

Embil JM, Iaconis J, Soto N, et al. 2005. Complicated skin and skin structure infections (cSSSI) in diabetic patients: bacteriology and clinical efficacy of meropenem (MEM) and imipenem-cilastatin (IPM) [abstract]. 45th Annual Meeting of the American Society for Microbiology, Washington, DC. 16-19 December. Abstracts of the 45th Interscience Conference on Antimicrobial Agents and Chemotherapy, 2306.

Eron LJ, Lipsky BA, Low DE, et al. 2003. Managing skin and soft tissue infections; expert panel recommendations on key decision points. $J$ Antimicrob Chemother, 52(Suppl. S1):i3-i17.

Fabian TC, File TM Jr, Embil JM, et al. 2005. Meropenem versus imipenem-cilastatin for the treatment of hospitalized patients with complicated skin and skin structure infections: results of a multicenter, randomized, double-blind comparative study. Surg Infect, 6:269-82.

Falagas ME, Barefoot L, Griffith J, et al. 1996. Risk factors leading to clinical failure in the treatment of intra-abdominal or skin/soft tissue infections. Eur J Clin Microbiol Infect Dis, 15:913-21.

Fish DN, Singletary TJ. 1997. Meropenem: a new carbapenem antibiotic. Pharmacother, 17:644-69.

Fridkin SK, Hagerman JC, Morrison M, et al. 2005. Methicillin-resistant Staphylococcus aureus disease in three communities. $N$ Engl J Med, 352:1436-44.

Fuchs PC, Barry AL, Brown SD. 2001. In vitro activities of ertapenem (MK-0826) against clinical bacterial isolates from 11 North American medical centers. Antimicrob Agents Chemother, 45:1915-18.

Fung HB, Chang JY, Kuczynski S. 2003. A practical guide to the treatment of complicated skin and soft tissue infections. Drugs, 63:1459-80.

Garau J, Blanquer J, Cobo L, et al. 1997. Prospective, randomized, multicentre study of meropenem versus imipenem/cilastatin as empiric monotherapy in severe nosocomial infections. Eur J Clin Microbiol Infect Dis, 16:789-96. 
Garnacho-Montero J, Garcia-Garmendia JL, Barrero-Almodovar A, et al. 2003. Impact of adequate empiric antibiotic therapy on the outcome of patients admitted to the intensive care unit with sepsis. Crit Care Med, 31:2742-51.

Graham DR, Lucasti C, Malafaia O, et al. 2002. Ertapenem once daily versus piperacillin-tazobactam 4 times per day for treatment of complicated skin and skin-structure infections in adults: results of a prospective, randomized, double-blind multicenter study. Clin Inf Dis, 34:1460-8.

Grayson ML, Gibbons GW, Habershaw GM, et al. 1994. Use of ampicillin/ sulbactam versus imipenem/cilastatin in the treatment of limbthreatening foot infections in diabetic patients. Clin Infect Dis, 18:68393.

Hecht DW. 2004. Prevalence of antibiotic resistance in anaerobic bacteria: worrisome developments. Clin Infect Dis, 39:92-7.

Hutchison M, Faulkner KL, Turner PJ, et al. 1995. A compilation of meropenem tissue distribution data. J Antimicrob Chemother, 36(Suppl A):43-56.

Jodrá VM, Pérez CD, Soler LS, et al. 2006. Results of the Spanish national nosocomial infection surveillance network (VICONOS) for surgery patients from January 1997 through December 2003. Am J Infect Control, 34:134-41.

Jones ME, Schmitz FJ, Fluit AC, et al. 1999. Frequency of occurrence and antimicrobial susceptibility of bacterial pathogens associated with skin and soft tissue infections during 1997 from an international surveillance program. Eur J Clin Microbiol Infect Dis, 18:403-8.

Jones ME, Karlowsky JA, Draghi DC, et al. 2003. Epidemiology and antibiotic susceptibility patterns of pathogens isolated from skin and soft tissue infections in the USA and Europe: a guide to appropriate antimicrobial therapy. Int J Antimicrob Agents, 22:406-19.

Karlowsky JA, Draghi DC, Jones ME, et al. 2003. Surveillance for antimicrobial susceptibility among clinical isolates of Pseudomonas aeruginosa and Acinetobacter baumanii from hospitalized patients in the United States, 1998 to 2001. Antimicrob Agents Chemother, 47:1681-8.

Kollef MH, Sherman G, Ward S, et al. 1999. inadequate antimicrobial treatment of infections: a risk factor for hospital mortality among critically ill patients. Chest, 115:462-74.

Kuti JL, Nightingale CH, Nicolau DP. 2004. Optimizing pharmacodynamic target attainment using the MYSTIC antibiogram: data collected in North America in 2002. Antimicrob Agents Chemother, 48:2464-70.

Lami JL, Wilson SE, Hopkins JA. 1991. Adjunctive antimicrobials in surgery of soft tissue infections: evaluation of cephalosporins and carbapenems. Am Surg, 57:769-74.

Leibovici L, Shraga I, Drucker M, et al. 1998. The benefit of appropriate empirical antibiotic treatment in patients with bloodstream infection. J Intern Med, 244:379-86.

Lipsky BA, Berendt AR, Deery HG, et al. 2004. Diagnosis and treatment of diabetic foot infections. Clin Infect Dis, 39:885-910.

Lipsky BA, Armstrong DG, Citron DM, et al. 2005. Ertapenem versus piperacillin/tazobactam for diabetic foot infections (SIDESTEP): prospective, randomized, controlled, double-blinded, multicentre trial. Lancet, 366:1695-703.

Livermore DM, Carter MW, Bagel S, et al. 2001. In vitro activities of ertapenem (MK-0826) against recent clinical bacteria collected in Europe and Australia. Antimicrob Agents Chemother, 45:1860-7.

Luna CM, Vujacich P, Niederman MS, et al. 1997. Impact of BAL data on the therapy and outcome of ventilator-associated pneumonia. Chest, 111:676-85

Maglio D, Teng R, Thyrum PT, et al. 2003. Pharmacokinetic profile of meropenem, administered at 500 milligrams every 8 hours, in plasma and cantharidin-induced skin blister fluid. Antimicrob Agents Chemother, 47:1771-3.

Masterton RG, Kuti JL, Turner PJ, et al. 2005. The OPTAMA programme: utilizing MYSTIC (2002) to predict critical pharmacodynamic target attainment against nosocomial pathogens in Europe. $J$ Antimicrob Chemother, 55:71-7.
Merrem (meropenem) product package insert. 2005 (October). AstraZeneca Pharmaceuticals, Wilmington, Delaware, USA.

Mouton JW, Van Den Anker JN. 1995. Meropenem clinical pharmacokinetics. Clin Pharmacokinet, 28:275-86.

Mutnick AH, Rhomberg PR, Sader HS, et al. 2004. Antimicrobial usage and resistance trend relationships from the MYSTIC programme in North America (1999-2001). J Antimicrob Chemother, 53:290-6.

Neuhauser MM, Weinstein RA, Rydman R, et al. 2003. Antibiotic resistance among gram-negative bacilli in US intensive care units. Implications for fluoroquinolone use. JAMA, 289:885-8.

Nichols RL. 1999. Optimal treatment of complicated skin and skin structure infections. J Antimicrob Chemother, 44:19-23.

Nichols RL, Smith JW, Geckler RW, et al. 1995. Meropenem versus imipenem/cilastatin in the treatment of hospitalized patients with skin and soft tissue infections. South Med J, 88:397-404.

Norrby SR, Gildon KM. 1999. Safety profile of meropenem: a review of nearly 5,000 patients treated with meropenem. Scand J Infect Dis, $31: 3-10$

Ong CT, Kuti JL, Nicolau DP. 2005. Pharmacodynamic modeling of imipenem-cilastatin, meropenem, and piperacillin-tazobactam for empiric therapy of skin and soft tissue infections: a report from the OPTAMA program. Surg Infect, 6:419-26.

Paterson DL, Mulazimoglu L, Casellas JM, et al. 2000. Epidemiology of ciprofloxacin resistance and its relationship to extended-spectrum beta-lactamase production in Klebsiella pneumoniae isolates causing bacteremia. Clin Infect Dis, 30:473-8.

Paterson DL, Ko W-C, Von Gottberg A, et al. 2003. Antibiotic therapy for Klebsiella pneumoniae bacteremia: implications of production of extended-spectrum -lactamases. Clin Infect Dis, 39:31-7.

Pellizzer G, Strazzabosco M, Presi S, et al. 2001. Deep tissue biopsy vs. superficial swab culture monitoring in the microbiological assessment of limb-threatening diabetic foot infection. Diabet Med, 18:822-7.

Pfaller MA, Segreti J. 2006. Overview of the epidemiological profile and laboratory detection of extended-spectrum-lactamases. Clin Infect Dis, 42(Suppl 4):S153-63.

Quale J, Bratu S, Gupta J, et al. 2006. Interplay of efflux system, ampC, and oprD expression in carbapenem resistance of Pseudomonas aeruginosa clinical isolates. Antimicrob Agents Chemother, 50:1633-41.

Raghavan M, Linden PK. 2004. Newer treatment options for skin and soft tissue infections. Drugs, 64:1621-42.

Red Book. 2005. Medical Economics Company, Inc. Montvale, NJ, USA.

Rello J, Ausina V, Ricart M, et al. 1993. Impact of previous antimicrobial therapy on the etiology and outcome of ventilator-associated pneumonia. Chest, 104:1230-5.

Rennie RP, Jones RN, Mutnick AH, et al. 2003. Occurrence and antimicrobial susceptibility patterns of pathogens isolated from skin and soft tissue infections: report from the SENTRY Antimicrobial Surveillance Program (United States and Canada, 2000). Diagn Microbiol Infect Dis, 45:287-93.

Rhomberg PR, Fritsche TR, Sader HS, et al. 2005. Comparative antimicrobial potency of meropenem tested against Gram-negative bacilli: report from the MYSTIC Surveillance Program in the United States (2004). J Chemother, 17:459-69.

Sader HS, Jones RN, Silva JB, et al. 2002. Skin and soft tissue infections in Latin American medical centers: four-year assessment of the pathogen frequency and antimicrobial susceptibility patterns. Diagn Microbiol Infect Dis, 44:281-8.

Stevens DL, Bisno AL, Chambers HF, et al. 2005. Practice guidelines for the diagnosis and management of skin and soft-tissue infections. Clin Infect Dis, 41:1373-406.

Swartz MN. 2000. Cellulitis and subcutaneous tissue infections. In: Mandell GL, Bennett JE, Dolin R (eds). Principles and practice of infectious diseases. 5th ed. New York: Churchill Livingstone. p 1037-57.

Tan JS, Wishnow RM, Talan DA, et al. 1993. Treatment of hospitalized patients with complicated skin and skin structure infections: doubleblind, randomized, multicenter study of piperacillin-tazobactam versus ticarcillin-clavulanate. Antimicrob Agents Chemother, 37:1580-6. 
Tassler H, Cullmann W, Elhardt D. 1993. Therapy of soft tissue infections with piperacillin/tazobactam. $J$ Antimicrob Chemother, 31(Suppl A): $105-12$

Teppler H, Gesser RM, Friedland IR, et al. 2004. Safety and tolerability of ertapenem. J Antimicrob Chemother, 53(Suppl S2):ii75-ii81.

Turnidge JD. 1998. The pharmacodynamics of beta-lactams. Clin Infect Dis, 27:10-22.

Vinh DC, Embil JM. 2005. Rapidly progressive soft tissue infections. Lancet Infect Dis, 5:501-13.

Wilcox MH. 2005. Efficacy of tigecycline in complicated skin and skin structure infections and complicated intra-abdominal infections. $J$ Chemother, 17 (Suppl 1):23-9.
Wilson MA. 2003. Skin and soft-tissue infections: impact of resistant grampositive bacteria. Am J Surg, 186 (Suppl 5A):35S-41S.

Wiseman LR, Wagstaff AJ, Brogden RN, et al. 1995. Meropenem: a review of its antibacterial activity, pharmacokinetic properties, and clinical efficacy. Drugs, 50:73-101.

Zervos MJ, Hershberger E, Nicolau DP, et al. 2003. Relationship between fluoroquinolone use and changes in susceptibility to fluoroquinolones of selected pathogens in 10 United States teaching hospitals, 19912000. Clin Infect Dis, 37:1643-8. 
